\title{
Diagnostic Utility of Haematological Scoring System (HSS) with Clinicopathological and Bacteriological Evaluation in Early Diagnosis of Neonatal Sepsis
}

\author{
Bhagyashree M. Ahirrao, Nandkumar V. Dravid, Mahesh H. Ahirrao, Arundhati S. Gadre and Shirish Gondane \\ Department of Pathology, ACPM Medical college Morane, Dhule, Maharashtra, India
}

\begin{abstract}
Background: Neonatal sepsis is a major cause of mortality with high incidence of 11 to 24.5 per 1000 live births in India. Delay in diagnosis further increases the mortality to $30-40 \%$ of total neonatal deaths. Though blood culture is gold standard for diagnosis of neonatal sepsis, it has limitations. The comprehensive Hematologic scoring system (HSS) formulated by Rodwell et al is used for early diagnosis of sepsis. This comprises of Immature/Total neutrophil ratio, Total PMN count, Immature/Mature ratio, Immature PMN count, degenerative changes in PMN, platelet count.

Methods: This is a prospective study of haematologic profiles of 303 neonates All neonates with congenital abnormalities diagnosed at birth admitted in neonatal care unit in our institute. Field stained blood smears were examined for HSS. Blood culture was done before administration of antibiotic treatment. Neonates with predisposing perinatal risk factors or if there was clinical suspicion of sepsis were included in this study from Nov 2014 to feb 2016.
\end{abstract}

Result: Of the 303 neonates in the present study, 77 had positive blood cultures, The incidence of septicemia was higher in males ( $72.7 \%$ ) than females $(27.3 \%)$. Majority of the neonates presented with early onset type of sepsis $(91 \%)$. Elevated I: T ratio and I:M ratio were seen in most cases of septicemia.

Conclusion: HSS is the most sensitive indicator of sepsis. Use of HSS by peripheral smear study Blood culture can be used effectively as a sepsis screen for early diagnosis is useful to reduce neonatal morbidity and mortality.

Keywords: Haematological Scoring System, Neonatal Sepsis, Peripheral Blood Smears

\section{Introduction}

Neonatal sepsis refers to a clinical syndrome characterised by systemic signs and symptoms due to generalised bacterial infection with a positive blood culture in the first 28 days of life. It is probably responsible for $30-50 \%$ of the total neonatal deaths each year. According to recent data from National Neonatal Perinatal Database (NNPD) 2002-03 collected from 18 centers from various parts of India, incidence of neonatal sepsis has been reported to be 29.9 per 1000 live births. ${ }^{[1]}$ Neonatal septicemia with its high incidence and grave prognosis, in spite of adequate treatment with modern antibiotics, has been a challenge for all times. Optimal diagnosis and treatment strategies are difficult to define. The signs and symptoms are protean with a high mortality and thus there is urgent need to know whether the baby has sepsis to institute treatment as quickly as possible.

Hematologic scoring system comprises of Immature/Total neutrophil ratio, Total PMN count, Immature/Mature ratio, Immature PMN count, degenerative changes in PMN, platelet count. ${ }^{[2]}$ Current study was undertaken to assess role of HSS in early diagnosis of neonatal sepsis with clinicopathological correlation.

\section{Materials and Methods}

The present study is a prospective analysis of the hematological profile of 303 neonates admitted to the neonatal intensive care unit at Tertiary care Medical College \& Hospital, during the period from November 2014 to February 2016. Neonates born at any gestational age with weight between 1000-2500gm with predisposing perinatal risk factors or if there was clinical suspicion of sepsis with clinical features of neonatal sepsis of IMCI criteria ( WHO 0ct2011) were included in this study. All neonates congenital abnormalities diagnosed at birth, mothers receiving antibiotic treatment regimen, neonates having clinical features resembling sepsis but attributable to causes other than sepsis like birth asphyxia, hypoglycaemia, inborn errors of metabolism, undergoing surgeries were excluded. A detailed clinical history of each patient was recorded after informed and written consent from parents.

Method of Collection of Data: The blood samples were sent to the Pathology laboratory in EDTA bulbs and had 
been collected by peripheral venipuncture using aseptic precautions. Neonates were divided into 3 groups :

Group 1 (Proven sepsis): neonates with sepsis (with positive blood culture)

Group 2 (Probable sepsis): neonates with probable infection (with clinical signs and negative blood culture)

Group 3 (No sepsis): normal neonates (without signs of sepsis).

The routine hematological investigations included hemoglobin, hematocrit, red blood cell indices (MCV, $\mathrm{MCH}$ and $\mathrm{MCHC}$ ), total WBC count, band cell count, I:T ratio, I:M ratio degenerative changes and platelet count. These investigations were performed on multichannel automated cell counter with standard calibration. Blood film was stained with Leishman's stained and. analysis of the smear findings were done by the pathologists blinded to the infection status of the neonate.

The WBC count was corrected for nucleated red cells and a differential count was performed manually. Immature neutrophils included promyelocyte, myelocyte, metamyelocyte and band forms.[fig:3] A band cell was defined as a neutrophil in which, the nucleus was indented by more than half, but in which the isthmus between the lobes was wide enough to reveal two distant margins with nuclear material between.[fig4] The polymorphonuclear leucocytes were also examined for degenerative morphological changes such as toxic granulation, toxic vacuolization and Dohle bodies.

The hematological findings were analyzed according to the hematological scoring system of Rodwell et al. which includes the following 7 findings-Total leucocyte count, total Neutrophil (PMN) count, Immature PMN count, Immature to Total PMN ratio (I:T), Immature to Mature PMN ration (I:M),Platelet count and Degenerative changes in neutrophils. [table 1,2]

C-reactive protein levels were also recorded. This test was done in the immunology laboratory by immunochromatography. Micro-ESR also noted.

For culture $2 \mathrm{ml}$ blood in were taken before administering any antibiotic and sent to lab immediately. Cultures were observed after 24-48hours \& 120 hours. If growth was observed, material was further analyzed for isolation of organism \& antibiotic sensitivity. If no growth was observed after 7 days, culture was reported negative.

Statistical Analysis: The data collected was statistically analyzed as per SPSS version 16, to find out the performance of the test individually and as a scoring system under the following parameters. Special emphasis was given to Sensitivity, Specificity, Positive predictive value, Negative predictive value. The Chi-Square Test was utilized to compare the observed and expected frequencies in each category. The contingency coefficient was used to measure the strength of association. This research work was approved by the institutional ethical committee.

\section{Result}

Age group wise maximum neonates i.e $79.9 \%$ were upto 24 hours of life, while $8.6 \%$ were 48 to 72 hours old and $6.6 \%$ were more than 96 hours of age. Of 303 neonates in the study confirmed sepsis was noted in $25.4 \%$ i.e 77 neonates having culture positive and clinical symptoms present, $53.1 \%$ i.e 161 neonates were labelled to be having probable sepsis because they ad negative culture reports but clinically positive symptoms. 65 neonates i.e $21.5 \%$ were labelled to be not having sepsis because they had culture and clinical negative findings.

In patients with $\geq 2500$ gms birth weight, $56.8 \%$ had probable sepsis and $16.9 \%$ had proven sepsis. Within 1500 to 2499 gms birth weight neonates, $34.8 \%$ had proven sepsis and $48.3 \%$ had probable sepsis. In 1000 to $1499 \mathrm{gms}$ birth weight neonates $58 \%$ had probable sepsis and $27.2 \%$ had proven sepsis. While in neonates with upto $1000 \mathrm{gms}$ birth weight $26.7 \%$ had either proven and probable sepsis. There was statistically significant $(p<0.01)$ difference of the sepsis status in different birth weight groups of neonates.

Of 15 neonates with less than 28 weeks gestational age $26.7 \%$ had proven or probable sepsis. Of 73 neonates with 28 to 32 weeks gestational age $21.9 \%$ had proven sepsis and $60.3 \%$ had probable sepsis. Of 15 neonates with 33 to 37 weeks gestational age $34.7 \%$ had proven and $50.5 \%$ had probable sepsis. Of 114 neonates with 38 weeks and above gestational age 19.3\% had proven sepsis and 54.4\% had probable sepsis. There was statistically significant $(p<0.05)$ difference of sepsis status in different gestational age groups of neonates.

Sepsis of proven or probable type was noted in 238 neonates in the study. Early onset sepsis was noted in 219 neonates and 19 had late onset sepsis. In proven sepsis neonates $90.9 \%$ had EOS while in probable sepsis also $92.5 \%$ had probable sepsis. Thus EOS was prominently noted in either of the sepsis status. In 189 neonates with preterm birth, $29.1 \%$ had proven sepsis and $52.4 \%$ had probable sepsis. While of 114 neonates with term birth $19.3 \%$ had developed proven sepsis and $54.4 \%$ had probable sepsis. There was statistically significant difference of Low birth weight $(p<0.05)$, very low birth weight $(p<0.05)$ and no significant difference ( $p>0.05)$ of mode of delivery, term of birth status of neonates with the sepsis status. 
Of 77 neonates being culture positive having proven sepsis, maximum i.e $41.6 \%$ had Klebsiella, 19.5\% - E coli and Staph aureus was isolated in $15.6 \%$. In 9.1\% neonates pseudomonas, $3.9 \%$ coagulase negative staphylococci microorganism was isolated while $2.6 \%$ either had Acinetobacter B or Enterococcus or $\mathrm{H}$ influenza or Proteus on culture examination.

Raised CRP level was notes in 144 neonates. Of 77 neonates with proven sepsis, 57.1\% had raised CRP.

Table 1: Hematologic Scoring System ${ }^{5}$.

\begin{tabular}{|l|l|l|}
\hline Criteria & Abnormality & score \\
\hline Total WBC count & $\begin{array}{l}\text { count } \leq 5,000 / \mu \mathrm{L} \\
\geq 25,000 \text { (at birth) } \\
\geq 30,000(12-24 \mathrm{hrs}) \\
\geq 21,000 \text { (day } 2 \text { onwards) }\end{array}$ & 1 \\
\hline Total PMN count & $\begin{array}{l}\text { No mature PMN seen } \\
\text { Increased/ decreased }\end{array}$ & 2 \\
\hline Immature PMN count & Increased & 1 \\
\hline I:T PMN ratio & Increased & 1 \\
\hline I:M PMN ratio & $\geq 0.3$ & 1 \\
\hline Degenerative changes in PMN & Toxic granules/cytoplasmicvacuoles & 1 \\
\hline Platelet count & $\leq 150,000 / \mu \mathrm{L}$ & 1 \\
\hline
\end{tabular}

The normal values are according to Manroe et al[3]:

Total PMN count-1800-5400/ul

Immature PMN count - 600/ul

Immature: Total PMN ratio- 0.12

Immature: Mature PMN ratio- $\geq 0.3$

Table 2: Interpretation of HSS.

\begin{tabular}{|c|c|}
\hline Score & Intrepretation \\
\hline$<2$ & Sepsis less likely \\
\hline $3-5$ & Sepsis probable \\
\hline$>5$ & Sepsis very likely \\
\hline
\end{tabular}

Table3: Distribution of neonates according to sex.

\begin{tabular}{|c|c|c|}
\hline Sex & Frequency & Percent \\
\hline Female & 115 & 38.0 \\
\hline Male & 188 & 62.0 \\
\hline Total & 303 & 100.0 \\
\hline
\end{tabular}

Table 4: Interpretation of HSS score $(n=303)$.

\begin{tabular}{|l|c|c|}
\hline Interpretation & Frequency & Percent \\
\hline Sepsis is unlikely (HSS $\leq 2)$ & 58 & 19.1 \\
\hline Sepsis is very likely (HSS 3or 4) & 153 & 50.5 \\
\hline Sepsis possible (HSS $\geq 5)$ & 92 & 30.4 \\
\hline Total & $\mathbf{3 0 3}$ & $\mathbf{1 0 0 . 0}$ \\
\hline
\end{tabular}

Of 161 neonates with probable sepsis $55.3 \%$ had raised CRP and in $16.9 \%$ with no sepsis CRP was raised. The difference of raised CRP in the three groups of sepsis status was statistically very highly significant $(p<0.001)$ [Table:4] For diagnosis of sepsis (Culture positive and Culture negative but clinical positive) the HSS component - Sensitivity, specificity, Positive and negative predictive value of HSS component in sepsis and probable $\operatorname{sepsis}(n=238)$. [ Table:5] 
Table 5: component in sepsis and probable sepsis $(\mathrm{n}=238)$ group.

\begin{tabular}{|c|c|c|c|c|c|}
\hline Sr.NO & HSS component & Sensitivity & Specificity & PPV & NPV \\
\hline 1 & Total WBC count & $21.8 \%$ & $98.5 \%$ & $98.1 \%$ & $25.6 \%$ \\
\hline 2 & Total PMN count & $85.7 \%$ & $23.1 \%$ & $80.3 \%$ & $30.6 \%$ \\
\hline 3 & Immature PMN & $88.2 \%$ & $18.5 \%$ & $79.8 \%$ & $30 \%$ \\
\hline 4 & Immature : Total PMN & $92.4 \%$ & $15.4 \%$ & $80 \%$ & $35.7 \%$ \\
\hline 5 & Immature : Mature PMN & $96.2 \%$ & $12.3 \%$ & $80.1 \%$ & $47.1 \%$ \\
\hline 6 & Degenerative Change & $49.6 \%$ & $92.3 \%$ & $95.9 \%$ & $33.3 \%$ \\
\hline 7 & Platelet count & $39.9 \%$ & $92.3 \%$ & $95 \%$ & $29.6 \%$ \\
\hline
\end{tabular}

Table 6: Relation of HSS score with sepsis category.

\begin{tabular}{|l|c|c|c|}
\hline Category & Score (0-2)\% & Score (3-4)\% & Score (>5)\% \\
\hline Sepsis & - & - & $77(100 \%)$ \\
\hline Probable sepsis & $36(22.4 \%)$ & $54(33.5 \%)$ & $71(44.1 \%)$ \\
\hline No sepsis & $22(33.8 \%)$ & $38(58.46)$ & $5(7.69 \%)$ \\
\hline
\end{tabular}

\section{Sepsis wise grouping of neonates}

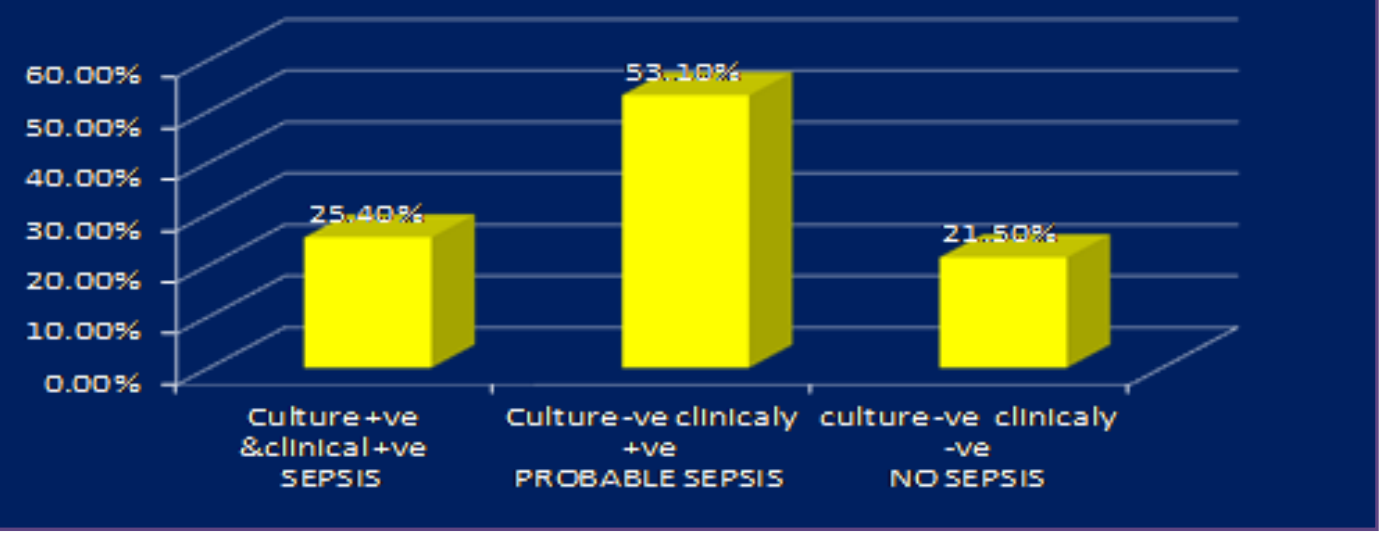

Fig.1: Grouping the neonates according to sepsis status $(\mathrm{n}=303)$.

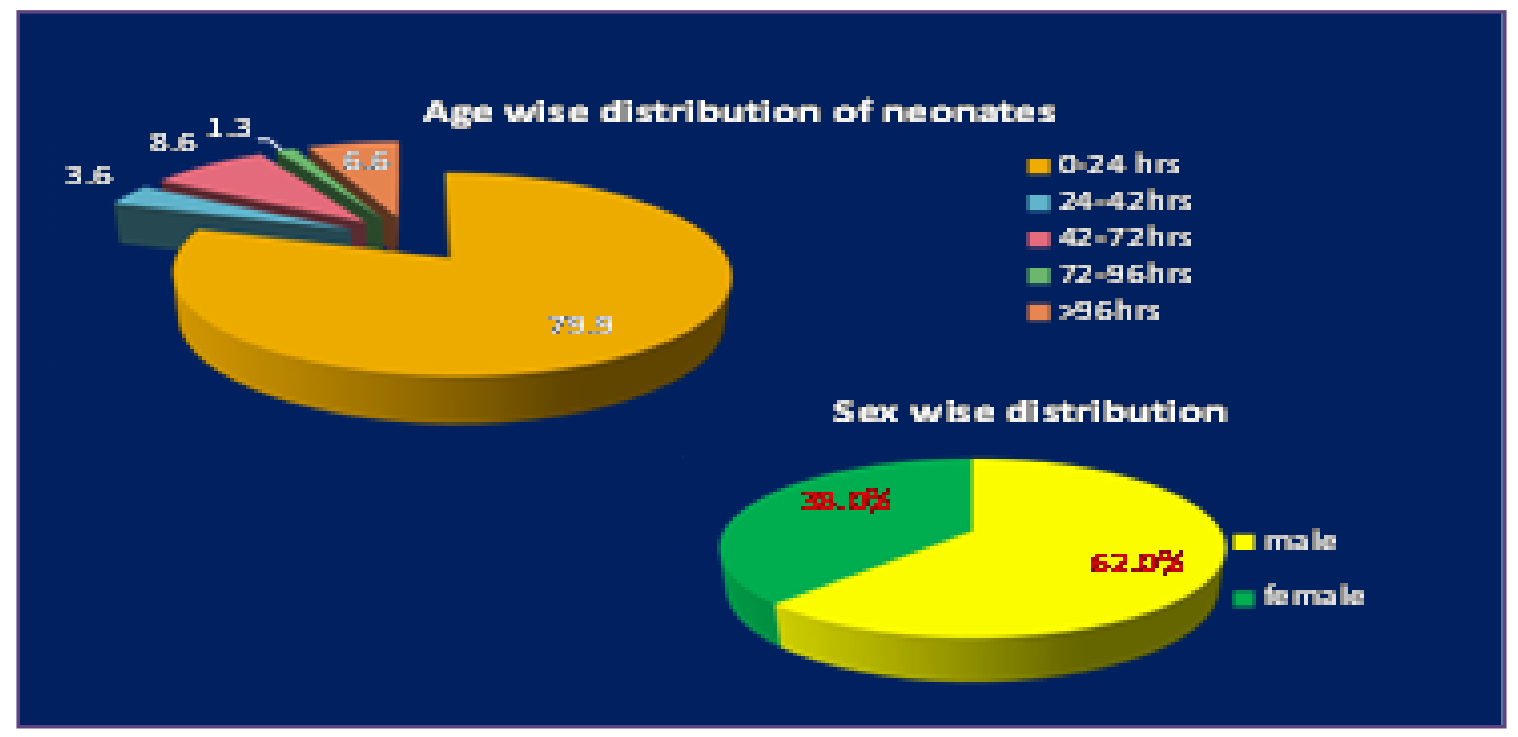

Fig. 2: Age group-wise distribution of neonates $(n=303)$.

Annals of Pathology and Laboratory Medicine, Vol. 4, Issue 6, November-December, 2017 


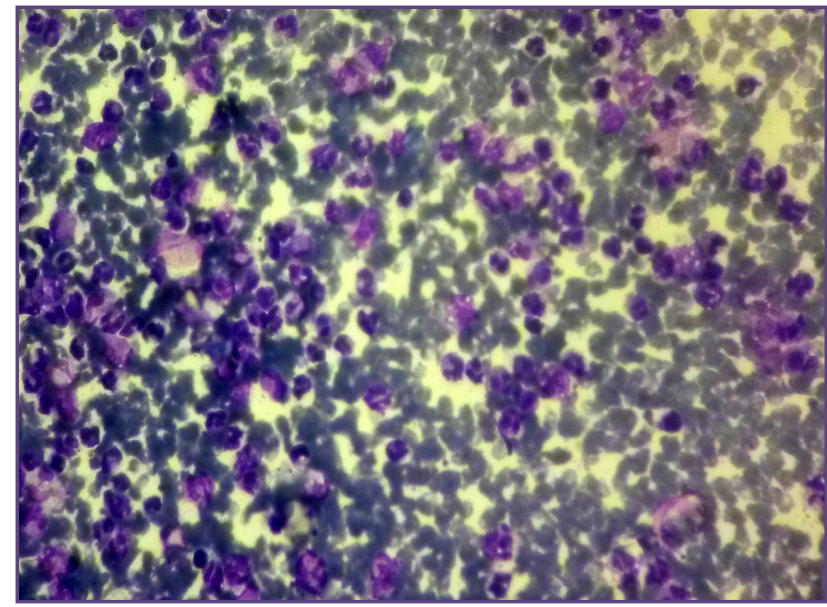

Fig. 3: Peripheral Blood Smear Showing Neutrophilic Leucocytosis Leishman's(x400).

\section{Discussion}

Sepsis is the commonest cause of neonatal mortality. It is responsible for about $30-50 \%$ of the total neonatal deaths in developing countries. It is estimated that up to $20 \%$ of neonates develop sepsis and approximately $1 \%$ die of sepsis related causes. ${ }^{[4]}$ The definite diagnosis of septicemia is made by a positive blood culture which requires a minimum period of $48-72 \mathrm{hrs}$ and yields positive result in $30-40 \%$ of cases. ${ }^{[5]}$

An early and accurate etiological diagnosis is not always easy, especially since the disease may start with minimal or non-specific symptoms. Delayed treatment until clinical recognition of signs and symptoms of sepsis entails risk of preventable mortality, notwithstanding the fact that presumptive antibiotic therapy may result in overtreatment.

In order to diagnose septicemia early, several rapid diagnostic tests have been described, which are easily performed and have the benefit of quick availability of reports. ${ }^{[5]}$ [Table: 6]

In the present study, score of $>3$ detected 202 out of 238 cases $(84.9 \%)$ of culture positive proven sepsis and probable sepsis. Rodwell study ${ }^{[2]}$ detected around $88 \%$ of neonates with sepsis and probable sepsis. Makkar et a ${ }^{[6]}$ found $93.7 \%$, of cases with microbiological and clinical evidence of sepsis. 36 out of 58 neonates had clinical evidence of sepsis though they had low HSS score. Thus high HSS score was more reliable predictor of sepsis than a low HSS score suggesting absence of disease. In the present study, I:T, I:M ratio has high sensitivity and specificity, high PPV and NPV comparable to many other studies ${ }^{[5,6,7,8,9,10,11]}$

Thrombocytopenia has poor sensitivity but high specificity. It is frequently associated with sepsis and indicates poor prognosis. This is due to increased platelet destruction,

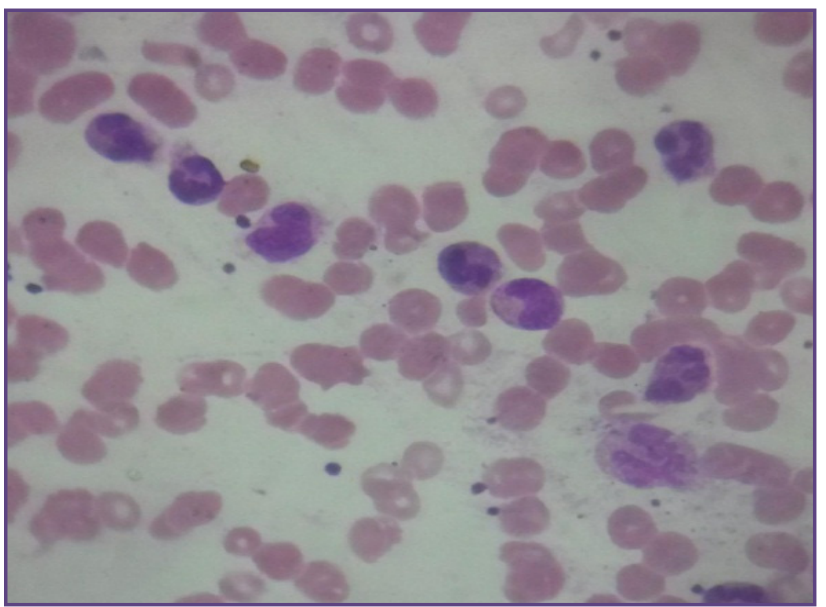

Fig. 4: Leishman's Stain (X100) Showing Immature Neutrophils.

sequestration secondary to infections and decreased production secondary to bone marrow suppression. Total WBC count has a low predictive value because of a wide range of normal count from $5000-20000 / \mathrm{mm} 3$. In Rodwell's study ${ }^{[2]}$ a score of $\geq 3$ identified 26 of 27 neonates in the sepsis group, this compared to the present study where it identified all 77 culture positive neonates. A score of $\geq 3$ was predictive of sepsis and more reliable indicator of sepsis than the best performing single parameter, the I:M ratio. Thus HSS, which is a combination of 7 parameters, is more diagnostic then any single parameter.

The higher the score, the greater was the certainty of sepsis being present. In Rodwell's study and the current study, $85-87 \%$ of positive cases had score of $\geq 4$. A score of $\geq$ 5 was even more predictive of sepsis. With a score which ranged between 0-2 there was a 99\% probability that sepsis was absent. The HSS should improve the efficiency of the $\mathrm{CBC}$ as a screening test for sepsis and permits an objective assessment of hematological changes. Ghosh et $\mathrm{al}^{[6]}$ analysed the hematological profiles in the light of the HSS and found that I:M ratio was the most reliable indicator in identifying infants with sepsis. The present study also showed that I:M ratio was most reliable.

\section{Conclusion}

The HSS is simpler, quick, cost effective and readily available tool in the early diagnosis of neonatal sepsis and could provide a guideline to decision regarding antibiotic therapy. We preferred to study efficacy of HSS alone as other parameters are costly to perform and require high technical lab support which would not have been relevant to rural setup like ours. Instead, a simple peripheral smear based test like HSS, which can distinguish between infected and non infected neonates with little laboratory backup is indeed a boon for peripheral pathologists and neonatologists. 


\section{Reference}

1. National Neonatal Perinatal Database 2002-2003 report. http://www.nnfi.org/images/NNPD2002-03.pdf. (Last accessed on Jan 22, 2016).

2. Rodwell RL, Leslie AL, Tudehope DI. Early diagnosis of neonatal sepsis using a Haematologic Scoring System. J Pediatr 1988;112:761-767.

3. Manroe BL, Weinberg AG, Rosenfeld CR, Browne R. The neonatal blood count in health and disease. Reference values for neutrophilic cells. J Pediatr 1979;95(1):89-98.

4. Shankar MJ, Agrawal R, Deorari AK, Paul V. Sepsis in newborn. AIIMSNICU protocols 2008:1-18.

5. Chandna A, Rao MN, Srinivas M, Shyamala S. Rapid Diagnostic Tests in Neonatal Septicemia. Indian J Pediatr 1988;55:947-953.

6. Ghosh S, Mittal M, Jaganathan G. Early diagnosis of neonatal sepsis using a hematological scoring system. Indian J Med Sci 2001;55:495-500.
7. Makkar M, Gupta C, Pathak R, Garg S, Mahajan NC Performance of evaluation of hematological scoring system in early onset neonatal sepsis. Journal of Clinical Neonatology 2013 Jan-Mar;2(1):25-29.

8. Supreetha MS et al. Evaluation of Neonatal septicemia using Haematological parameters. International Journal of Recent and Scientific Research vol 6,Issue 2,pp 2775-2778,Feb 2015.

9. Narasimha A, Harendrakumar ML. Significance of hematological scoringsystem (HSS) in early diagnosis of neonatal sepsis. Indian $\mathrm{J}$ Hematol Blood Transfus 2011;27(1):14-17.

10. Gheibi S, Fakoor Z, Karamyyar M, Khashabi J, Ilkhanizadeh B, Sana FA, et al. Coagulase negative staphylococcus; most common causes of neonatal septicemia in Urmia, Iran. Iran J Pediatr 2008 Sept;18(3):237-243.

11. Khair KB, Rahman MA, Sultana T, Roy CK, Rahman MQ, Shahidullah $\mathrm{M}$ et al. Role of hematological scoring system in early diagnoses of neonatal septicemia. BSMMU J 2010;3(2):62-67

*Corresponding author:

Dr. Bhagyashree Ahirrao, Department of Pathology, ACPM Medical college Morane, Dhule, Maharashtra, India

Phone: +91 9823959415

Email: dr.bhagyashreeahirrao@gmail.com

Date of Submission : 06.05.2017

Date of Acceptance : 19.09.2017

Financial or other Competing Interests: None.

Date of Publication : 21.12.2017 